\title{
"La arqueología se ha convertido en un mero trámite incómodo en los proyectos de rehabilitación urbana"
}

El desmantelamiento de un torreón de origen medieval para su posterior reconstrucción, como parte del proyecto de escalera mecánica del palacio de congresos de Toledo, recientemente inaugurada, ha puesto de manifiesto el escaso peso que el patrimonio sigue teniendo en el desarrollo urbanístico de la ciudad, que ostenta el título de patrimonio mundial. La torre era el único resto de la muralla que cerraba el espacio comprendido entre la puerta de Alcántara y la desparecida puerta de los Grederos. Lejos de ser una muestra de respeto al patrimonio es un desprecio a su esencia, opina el arqueólogo Ruiz Taboada.

Arturo Ruiz Taboada | arqueólogo

URL de la contribución <www.iaph.es/revistaph/index.php/revistaph/article/view/3487>

\section{Introducción}

Toledo se muestra ideal para poner en práctica proyectos y diseños arquitectónicos imposibles, conviviendo o compitiendo con las arquitecturas medievales que han hecho de la ciudad una de las más conocidas del mundo. En el resto de España la situación no es muy distinta. Asistimos a multitud de intervenciones en cascos históricos o monumentos que, cuanto menos, obligan a reflexionar sobre la gestión de aquello que hemos heredado de nuestros padres y lo que queremos dejar a nuestros hijos (TORQUEMADA, 2010). Las actuales leyes adolecen de la suficiente ingenuidad como para dejarse influir por intereses públicos y privados, que se sirven de conceptos como modernidad, renovación o vanguardia para imponer sus criterios a la hora de intervenir en patrimonio.

Por regla general, estas intervenciones suelen considerar al bien como algo mejorable, obsoleto o ajeno a la evolución "lógica" que experimentan los contextos urbanos o rurales en los que se encuentran. Mientras que en otras disciplinas como la pintura hubiera sido impensable sustituir una de las meninas por otra pintada por Picasso, en arquitectura no sólo se justifican similares inferencias en el patrimonio, sino que se las considera como enriquecedoras y necesarias. Pese a ello, no es lo mismo intervenir en un monumento para consolidarlo y evitar su deterioro que, sirviéndose de esa intención, transformarlo o adaptarlo a preceptos arquitectónicos contemporáneos, cuanto no demolerlo.
El torreón de Gerardo Lobo tuvo la desgracia de encontrarse en la trayectoria de la escalera mecánica de acceso al nuevo palacio de congresos de la ciudad, lo que ha derivado en su demolición para su posterior reconstrucción, una vez finalizadas las obras. El desmantelamiento piedra a piedra del torreón ha contado en todo momento con la autorización de la administración competente. Lo que en cualquier parte del mundo la acción hubiera sido catalogada como destrucción o saqueo, aquí empleamos un montón de eufemismos para legitimarla: traslado programado, desmonte técnico, derribo con control arqueológico, etc. No importa la normativa en materia de patrimonio que se encuentre en vigor en cada época, el resultado parece ser siempre el mismo.

Casos como el de la plaza de Oriente de Madrid, el edificio de la plaza de Santa Teresa de Ávila (FERNÁNDEZ SALINAS, 2009). el parador de Lorca o el teatro romano de Sagunto (ALMAGRO, 1993), forman ya parte de una tradición consolidada de gestión de patrimonio, que nos aleja día a día de aquella realizada en otros países como Italia, Inglaterra o Francia. En este sentido, las constantes revisiones a las que se ven sometidas las actuales leyes de patrimonio que tutelan la conservación de este tipo de restos no acaban de garantizar su conservación y puesta en valor.

Toledo se está convirtiendo en referente a la hora de determinar lo que no se debe hacer en este tipo de ciudades históricas. Su ingente patrimonio hace que las 

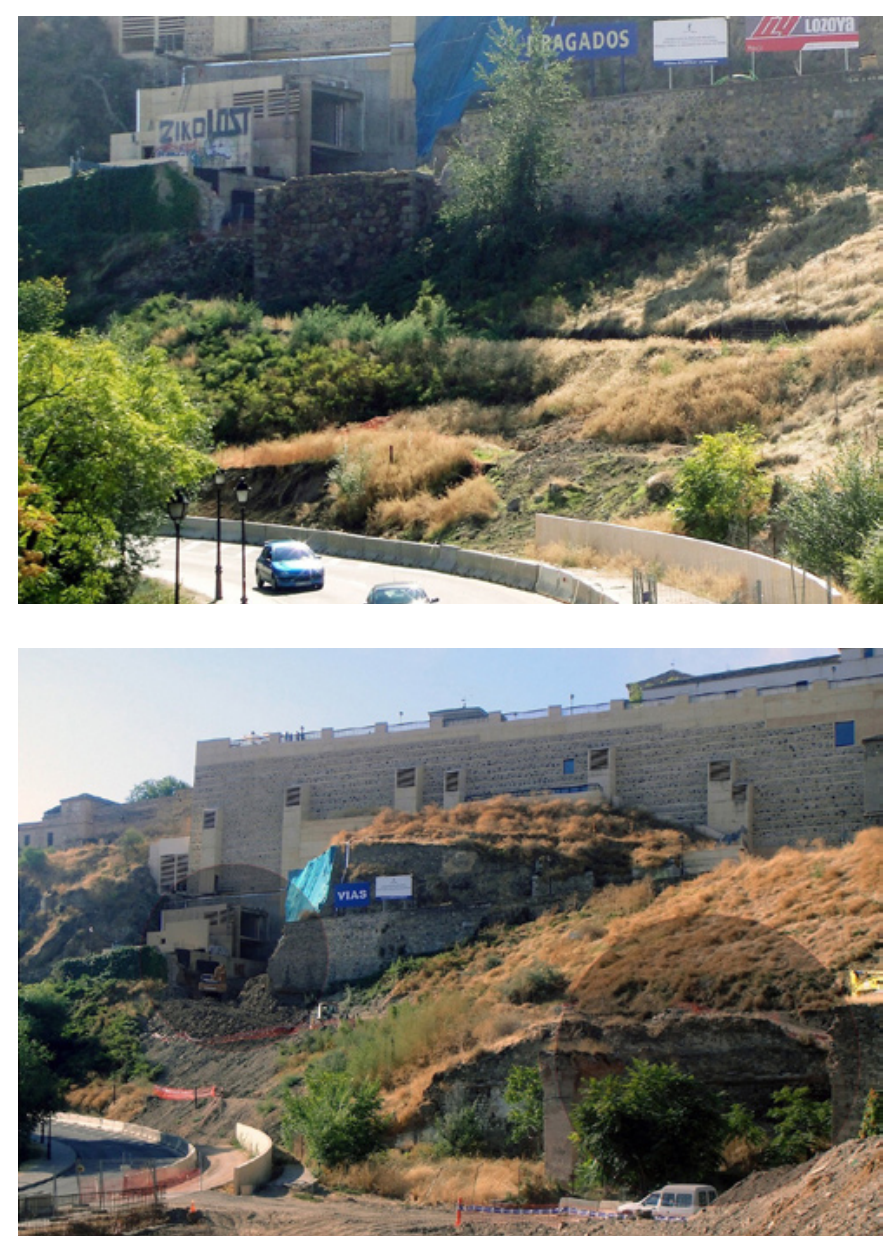

Desarrollo de las obras de demolición del torreón y su relación con las ruinas del convento de San Pablo (2012-2013) | fotos Arturo Ruiz Taboada

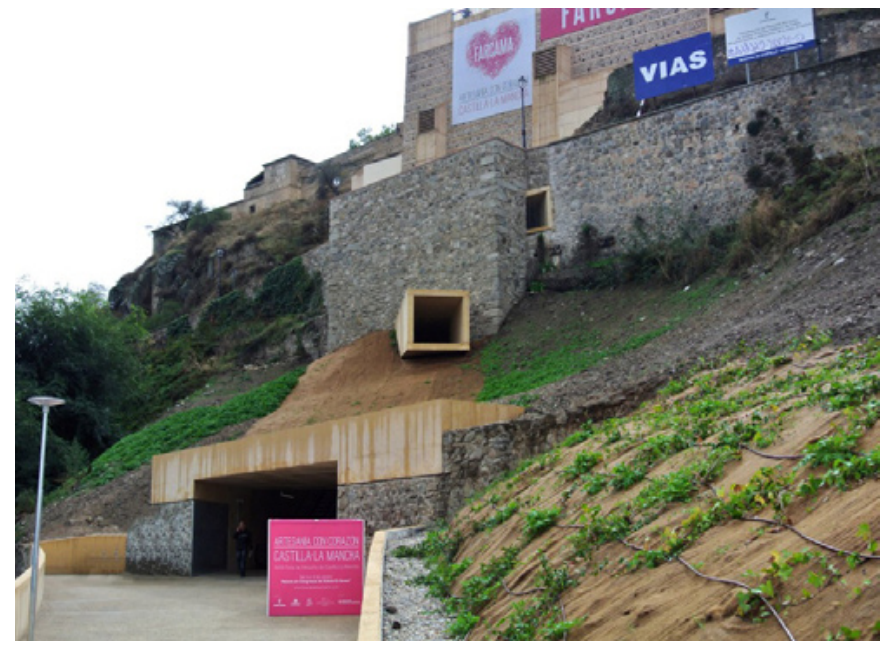

Estado actual del torreón reconstruido | foto Arturo Ruiz Taboada administraciones no sepan como gestionarlo y que el habitante no lo valore en su justa medida. Como ejemplo, los nuevos descubrimientos arqueológicos que se producen a diario pasan desapercibidos y, lejos de considerarlos como una fuente de conocimiento y riqueza, son vistos como un impedimento al desarrollo económico. El toledano se muestra complaciente con lo que tiene, suficiente de cara al turismo al ser fuente "inagotable" de ingresos. Frente a lo que ocurre en otras ciudades patrimonio mundial como Mérida, en Toledo, cualquier resto arqueológico que se descubre se vuelve molesto y sirve como arma arrojadiza para justificar los intereses de unos y otros (RUIZ TABOADA, 2012).

Lejos de encontrar un equilibrio entre la conservación y puesta en valor del patrimonio y el desarrollo y modernización de la ciudad, la mayoría de los proyectos que se plantean carecen de un ideario de ciudad histórica. Prueba de ello son las recientes actuaciones en el circo romano, financiadas con el $1 \%$ cultural, para la reorganización del tráfico y aparcamiento en su interior; la reconstrucción de la muralla del paseo de Recaredo, posterior al colapso consecuencia de las obras de construcción de una escalera mecánica en esa zona (RUIZ TABOADA, 2012), o la destrucción parcial de una necrópolis medieval con motivo de la puesta de la primera piedra del malogrado Quixote Crea (BARRIO ALDEA; RUIZ TABOADA; SÁNCHEZ PELÁEZ et ál., 2011).

La entrada en vigor del Plan Especial del Conjunto Histórico de Toledo (BUSQUETS MORENO, 1996), lejos de servir como referente para crear esta idea de ciudad, ha resultado ser una de las principales causas de su deterioro. El motivo es que únicamente se centra en aspectos urbanísticos y arquitectónicos, relegando a un segundo plano el contexto histórico de la ciudad. Al necesario concurso del estudio de arquitectura que lo redactó, le falta en un plano similar el complemento técnico que pudieran haber aportado arqueólogos e historiadores. El resultado desde su entrada en vigor hace dos décadas ha sido la progresiva destrucción de la identidad histórica de la urbe. Los nuevos diseños urbanísticos, amparados por este plan, parecen rivalizar con las arquitecturas medieval y moderna existentes (RUIZ TABOADA, 2012). 
Las intervenciones actuales, lejos de intentar respetar la esencia histórica de Toledo, pretenden dotar a los edificios de una firma contemporánea que, en muchos casos, recuerda a los grafiti en los monumentos clásicos, tan de moda entre los viajeros de finales del siglo XIX (RUIZ TABOADA, 2012). El inicio de la metamorfosis de la ciudad hay que situarlo en la década de los 50 del siglo pasado, en la que muchas manzanas de casas medievales fueron demolidas para construir viviendas de protección oficial. Esta tendencia ha continuado en la década de los 80 , con polémicas como la construcción del edificio de Chueca Goitia o la Consejería de Agricultura y, en la década de 2000, con proyectos de urbanización como Vega Baja (MARTÍNEZ CITORES, 2008), la ampliación del Alcázar para albergar el Museo del Ejército o intervenciones controvertidas en monumentos como la torre del Salvador o la iglesia del Cristo de la Luz (RUIZ BALLESTEROS, 2009). En la actualidad, esta progresión resulta imparable y está contribuyendo a que se desvanezca la esencia histórica de una ciudad única en el mundo.

\section{El torreón de la calle Gerardo Lobo}

Con motivo de la construcción de una escalera mecánica que comunica el barrio de la estación de autobuses de Toledo con el palacio de congresos, obra de Moneo, se ha desmantelado el único torreón que daba constancia de la muralla medieval de esa parte de la ciudad. Aunque el proyecto contemplaba su reconstrucción posterior, integrado en la nueva escalera proyectada, generando una anastilosis difícilmente entendible, el daño es ya irreparable. Las obras de la escalera no sólo han afectado a este torreón, sino a las cercanas ruinas del convento de San Pablo. La urbanización del entorno pretende integrar estas ruinas sin que, por desgracia, se haya tenido en cuenta ni el impacto de la maquinaria pesada empleada, o la resistencia estructural de los restos ante los nuevos taludes y caminos diseñados.

Aunque el arqueólogo responsable asegura que se trata de una construcción de finales del siglo XIX (La Tribuna $31 / 08 / 13$ ), lo cierto es que su origen se remonta a la Alta Edad Media. El sector de muralla al que pertenece se localiza entre la puerta de Alcántara y la desaparecida puerta de Los Grederos, junto al convento de San Pablo.
Tras la reconquista de la ciudad por el rey Alfonso VI, las incursiones de almorávides y almohades obligaron a reforzar las defensas de este sector de la ciudad. No sólo se reconstruyeron los antiguos muros andalusíes, sino que se añadieron nuevas torres y puertas, como la cercana puerta del Vado (RUIZ TABOADA; FERNÁNDEZ DEL CERRO, 2009). A partir del siglo XIV, la muralla de esta zona empieza a perder su funcionalidad defensiva, entrando en un progresivo deterioro, hasta convertirse en la tapia que existe en la actualidad y que delimita la calle de Gerardo Lobo.

Las referencias documentales más antiguas de esta calle las encontramos en un plano de principios del XVIII realizado por Navia y conservado en el Archivo Municipal de Toledo. Las reconstrucciones historicistas a las que se ve sometido el torreón desde finales del siglo XIX y a lo largo del XX, de las que habla el arqueológico director de la obra, son el final y no el inicio de su historia.

Con respecto al torreón desmontado, no es difícil encontrar pruebas materiales de su milenaria historia. Ya aparece en el grabado de Anton Vanden Wyngaerde en 1563 y en el cuadro del Greco Vista y plano de Toledo pintado hacia 1610, así como en la cartografía del proyecto para hacer navegable el manzanares diseñado por Carlos y Fernando Grunnemberg de 1668. El mismo torreón también está recogido en el plano de Francisco Coello y Maximiano Hijón de 1858, varios años antes de la reestructuración del entorno del puente de Alcántara, tras la demolición de su patio de armas en 1864. La reorganización urbana de esta zona se hizo motivada por la mejora general de los accesos a la ciudad y el saneamiento de las arquitecturas en ruina.

Entre 1860 y 1880 fueron demolidos monumentos emblemáticos como el artilugio de Juanelo, el arco de Zocodover, la iglesia de San Isidoro o el mencionado patio de armas. En este contexto de modernización de accesos es donde se debe situar la última remodelación y sustitución del paramentos en mal estado de este torreón. Más de un siglo después, el derribo del torreón de Gerardo Lobo mantiene el espíritu de estas intervenciones decimonónicas, en las que se eliminaba aquello que estorbaba para la "modernización" de la ciudad. 


\section{El torreón en el marco de la nueva legislación de patrimonio de Castilla-La Mancha}

La demolición del torreón para su posterior reconstrucción en el mismo sitio vuelve a poner de actualidad la gestión del patrimonio en Toledo, declarada por la UNESCO patrimonio mundial. En los últimos años han sido muchas las voces que han mostrado su preocupación por la deriva que estaba tomando la intervención en sus restos históricos. Como hemos visto, lo ocurrido con este torreón forma parte de una larga lista de actuaciones controvertidas en lugares emblemáticos de la ciudad, en los que los intereses urbanísticos siempre han primado frente a los patrimoniales.

La escasa relevancia que se da al monumento en el marco de los proyectos de rehabilitación ha convertido a la arqueología en un mero trámite administrativo, incómodo e irrelevante, aunque imprescindible para validar estos proyectos. El torreón de Gerardo Lobo es buena muestra de ello; independientemente de su antigüedad, lo realmente preocupante es que en el diseño de la escalera mecánica proyectada no se haya considerado la conservación de esta emblemática torre. Parece ser que resulta más sencillo desmantelar la estructura para luego reconstruirla que desplazar el trazado de la escalera para conservarla. Este tipo de planteamientos hace que la arqueología sólo se considere para justificar legalmente este tipo de acciones. En este sentido, la sociedad, las instituciones, los responsables de urbanismo, arqueólogos y arquitectos, deben plantearse el futuro que quieren dar a su pasado. Hasta que esto ocurra, la ciudad seguirá completamente desprotegida.

Así, se está empezando timidamente a cuestionar la eficacia de un Plan Especial para el Casco Histórico de Toledo, que no tiene en consideración el valor ambiental, material e histórico de la ciudad y que, desde su entrada en vigor, no ha hecho otra cosa que transformar irreversiblemente su imagen y personalidad. Si a esto sumamos que las instituciones responsables de la gestión del patrimonio toledano aún consideran a disciplinas como la arqueología un freno a la modernización de la ciudad, el panorama resulta del todo desolador. Estas instituciones, cuyo fin principal es velar por la conservación y racionalización de las rehabilitaciones, aún no han encontrado el sentido de su propia existencia y, en la mayoría de los casos, se encuentran a merced de las modas y tendencias que, mal entendidas, están contribuyendo al deterioro de la ciudad. La recientemente creada escuela de arquitectura en el campus de la Fábrica de Armas de Toledo, lejos de reflexionar sobre esta problemática, se sirve de la ciudad como un laboratorio en el que poder experimentar nuevas tendencias, analizando el monumento únicamente desde la perspectiva del arquitecto. Su programa de estudios carece de una formación humanística complementaria, impartida por historiadores del arte y arqueólogos, que contribuya a que futuros arquitectos aprendan a hacer otra lectura histórica de la ciudad y de sus monumentos.

En este contexto, en mayo de 2013, el Diario Oficial de Castilla-La Mancha publicaba la Ley 4/2013 de Patrimonio Cultural de la región que sustituía a la 4/1990, primera ley de este tipo en ser aprobada por una comunidad autónoma. Esta última, a su vez, sustituía a la 16/1985 de ámbito nacional. Frente a lo que ha ocurrido en la vecina Madrid con una reforma similar, la nueva Ley ha contado con un amplio consenso en la región. Como señala Benítez de Lugo (2013), las críticas vertidas sobre el texto madrileño (desprotección del patrimonio, desmantelamiento de un modelo eficaz de gestión, etc.) no son extrapolables al caso castellano manchego. La nueva norma de Castilla-La Mancha es un instrumento moderno, innovador y que supone una clara mejoría con respecto a la situación existente en legislaturas anteriores.

No obstante, este tipo de leyes sigue sin dar con la fórmula que permita combinar las actuaciones arquitectónicas sobre el patrimonio con el respeto al mismo. Su escasa efectividad de este tipo de leyes viene dada tanto por la gran ambigüedad del reglamento que las rige, como por la falta de criterio de la administración a la hora de aplicar modelos de actuación en las obras de rehabilitación.

Por su situación dentro del casco histórico de Toledo, el torreón demolido se encontraba condicionado a un estudio arqueológico previo, según se recoge en esta nueva ley, y que se hizo cumpliendo todos los precep- 
tos de la misma. En su artículo 27, punto primero, se define claramente el tipo de intervención a desarrollar al requerir "autorización previa de la Consejería competente en materia de Patrimonio Cultural, que contendrá las condiciones y plazos de ejecución de dicha intervención". Esta autorización deberá tener carácter previo a la concesión de la correspondiente licencia municipal y obliga al promotor a aportar un informe arqueológico sobre el bien que se pretende intervenir. Este informe debe recoger una propuesta de actuación integral y de carácter multidisciplinar, de acuerdo con los criterios de un equipo técnico cuya composición estará determinada por las características del bien y el tipo de intervención a llevar a cabo. En este sentido, se hace especial incidencia en la necesidad de evaluar tanto los efectos de la intervención arquitectónica como en el diseño de un programa de mantenimiento y conservación. A la vista de dicho estudio, la consejería competente en materia de patrimonio cultural podrá autorizar la obra y, en su caso, establecerá los condicionantes que deberán ser incorporados al proyecto de intervención, en su caso.

Lo cierto es que la puesta en práctica de esta normativa supone que cualquier intervención en el patrimonio esté sometida a la total subjetividad de la administración competente a la hora de evaluar la importancia del objeto a intervenir. Siendo conscientes de que la gestión del patrimonio no puede estar anclada en el inmovilismo y la conservación a ultranza, leyes como la actualmente aprobada no dejan de ser instrumentos para que, en según que momentos, se pueda "flexibilizar" la gestión de este tipo de bienes.

Asumida así la legalidad de la intervención en el torreón, se hace imprescindible reflexionar sobre su idoneidad o no, ya que ha sorprendido a muchos ciudadanos -sobre todo a los constructores y promotores privados- y a algunos arqueólogos conocedores del medio toledano. Los primeros consideran que mientras deben adaptar sus proyectos a los hallazgos arqueológicos confirmados en los trabajos por ellos financiados, la administración puede permitirse el lujo de desmontar o reubicar una pieza sin ningún tipo de remilgo o sensibilidad referida a la conservación o ubicación. Algo que ni se hubiera permitido en casos similares, que fueron tratados de manera muy diferente -piensan en alto los constructores- por el hecho de tratarse de actuaciones privadas. Los segundos ya no se sorprenden por algo que forma parte de su día a día profesional y ven como los monumentos deben adaptarse a los proyectos de arquitectura que se diseñan para ellos, cuando la lógica sugiere lo contrario. El caso de Gerardo Lobo, en el que se sustituye un torreón histórico por otro de nueva planta, lejos de ser una muestra de respeto al patrimonio es un desprecio a su esencia.

\section{BIBLIOGRAFÍA}

- Almagro, A, (1993) La "reconstrucción" del Teatro Romano de Sagunto. Reflexiones en torno a una polémica. Archivo Español de Arqueología, vol. 66, pp. 324-329

- BARRIO ALDEA, C.; RUIZ TABOADA, A.; SÁNCHEZ PELÁEZ, E. et ál. (2011) Destrucción parcial de la necrópolis medieval de Toledo. Restauro, n. ${ }^{\circ} 10$, pp. 28-33

- BENITEZ DE LUGO ENRICH, L. (2013) La protección del patrimonio arqueológico en Castilla-La Mancha. Reflexiones sobre la Ley 4/2013 de patrimonio cultural. revista ph, Panorama, n. ${ }^{\circ}$ 84, pp. 22-25

- BUSQUETS MORENO, C. (1996) Plan Especial del Conjunto Histórico. Toledo: Ayuntamiento de Toledo, Caja Castilla La Mancha y Empresa Municipal de la Vivienda, 1996

- FERNÁNDEZ SALINAS, V. (2009) El papel de ICOMOS en los procesos de declaración y seguimiento del Patrimonio Mundial. Patrimonio cultural de España, n. ${ }^{\circ} 2$, pp. 167-177

- MARTíNEZ CITORES, C. (2008) Vega Baja de Toledo: crónica de un rescate. [Toledo]: C. Martínez, 2008

- RUIZ BALLESTEROS, J. (2009) ¡Que lo tiren!. ABC [en línea], 10/12/2009 <http://www.abc.es/hemeroteca/historico10-12-2009/abc/Toledo/ique-lo-tiren!_1132446958089.html> [consulta: 09/10/2014]

- RUIZ TABOADA, A. (2012) Arquitectura residencial y religiosa, Toledo (ss. X a XVIII). Madrid: La Ergástula, 2012

- RUIZ TABOADA, A.; FERNÁNDEZ DEL CERRO, J. (2009) La puerta del Vado de Toledo. [Toledo]: Antonio Pareja, 2009

- TORQuemadA, B. (2010) Pifias monumentales. ABC [en línea], 10/08/2010, <http://www.abc.es/20100808/ espana/pifias-monumentales-201008081628.html> [consulta: 09/10/2014] 